\title{
EIMERIA CENTROCERCI N. SP. \\ DU CENTROCERCUS UROPHASIANUS (COQ DE BRUYÈRE) (1)
}

\section{Par Félix SIMON}

Au début de septembre 1932, la Commission du gibier du Wyoming a demandé au parasitologue, chargé des recherches à la Station agricole expérimentale de l'Université de Wyoming, d'étudier une épizootie qui sévissait sur les coqs de bruyère du comté de Frémont. Au milieu de cette épidémie, dans un champ de luzerne couvrant environ un mille carré, on avait estimé qu'environ 400 jeunes oiseaux, sur une population totale de 2.000 , étaient morts le 12 septembre. Les recherches montrèrent que cette mortalité élevée était due à une coccidie, identifiée plus tard comme Eimeria angusta Allen 1934. En même temps, une autre coccidie, décrite ici sous le nom d'E. centrocerci n. sp., existait en petit nombre.

En août 1938, plusieurs jeunes coqs de bruyère furent capturés le long du cours supérieur de la rivière Sweetwater, Wyoming. Ils ont fourni le matériel qui a servi à la description de cette nouvelle espèce et à des expériences d'infestation de jeunes poulets domestiques, expériences qui ont d'ailleurs échoué. Eimeria angusta existait aussi dans ce matériel.

Description. - Oocystes incolores, ellipsoïdes, mesurant $20 \mu, 75$, à $24 \mu, 90$ sur $16 \mu, 60$ à $17 \mu, 84$ (moyenne : $22 \mu, 61$ à $17 \mu, 14$ ). Sporocystes de $11 \mu, 20$ à $13 \mu, 28$ sur $6 \mu, 64$ à $7 \mu, 05$ (moyenne $11 \mu, 76$ sur $7 \mu, 63)$. Opercule présent, mais difficilement visible. Masse résiduelle des oocystes ayant l'aspect d'une très petite inclusion réfringente. Il y a aussi un corps résiduel sporocystique et des corps de Stieda.

Localisation : Caeca.

Hôte type : Centrocercus urophasianus (coq de bruyère).

Localité type : Bords de la rivière Sweetwater, Wyoming (U.S.A.).

(1) Traduit de l'anglais par le $\mathrm{D}^{\mathrm{r}}$ Maurice Langeron.

Annales de Parasitologie, T, XVII, No 2, - $1^{\text {er }}$ mars 1939 , p. 137-138. 
Discussion. - Parmi les coccidies d'oiseaux qui ont des oocystes elliptiques operculés, $E$. centrocerci diffère de $E$. lyruri et d'E. lagopodi par sa masse résiduelle et d'E. angusta par ses dimensions plus petites et son diamètre comparativement plus grand.

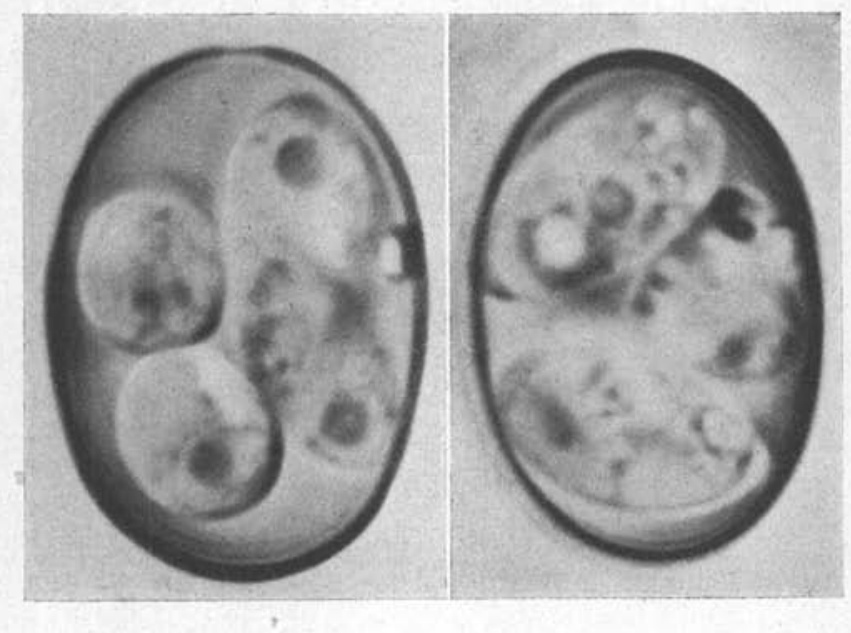

FIG. - Eimeria centrocerci n. sp.

\section{Bibliographie}

ALLEN (E. A.). - Eimeria angusta n. sp. and Eimeria bonasae n. sp. from grouse, with a key to the species of Eimeria in birds. Trans. amer. microse. Soc., LIII, 1934, p. 1-5.

Scott (J. W.) et Honess (R.). - On a serious outbreak of coccidiosis among sage chickens. Jl. Colo. Wyo. Acad. Sc., I, 1933.

University of Wyoming, Agricultural Experiment Station. 